question of the microscopy of the living eye and which persuaded me to publish this case are :

(I) The abundance of crystals and their localization on the anterior capsule, and as a result of this, the extraordinary play of light and colours.

(II) The instability of this phenomenon, which disappeared in a few months.

\title{
A SHORT STUDY OF THE COMPLICATIONS OF CATARACT EXTRACTION IN EGYPT WITH SOME PRACTICAL SUGGESTIONS
}

BY

\author{
H. PÉRETZ, M.D.(Montpellier)
}

CAIRO

Mr colleagues will agree with me that in Egypt cataract may be complicated by a great many other diseases of the eye. These may affect the globe or the eyelids, but whether they be primary or of a cicatricial nature, either may hinder the good progress of the operation or jeopardize a successful issue. It is, therefore, of the greatest importance for the surgeon to be well acquainted with such complications in order that he may be better able to overcome them when they present themselves.

After 20 years of careful observation, I have been able to point out to our young Egyptian colleagues the importance of these complications, but at the same time they ought to be brought to the knowledge even of European surgeons so that, by being better acquainted with tropical ophthalmology, they will not criticize us adversely if we do not follow exactly the usual technique for cataract extraction.

In the first place, it is of interest to compare the frequency of cataract in Egypt with that in other countries, for it might appear that it is a relatively rare condition in Egypt. About a quarter of a century ago, Dr. Osborne, of Alexandria, tried to ascertain the real facts, and in his paper* "Some Characteristic Observations of the Ophthalmic Surgeon in Egypt " he compared the statistics of operations performed at the clinique of Duke Charles Theodore, of Bavaria (Munich) with those done at the European Hospital (Alexandria). The figures examined covered the period 18981907 and whilst 35.9 per cent. of the operations performed at

* Osborne.-Bulletin of the Ophthalmological Society of Egypt, p. 56, 1908. 
Munich were found to be extractions for cataract, only $9 \cdot 8$ per cent. of the operations at Alexandria were for senile cataract.

In my opinion, however, the statistics of the two cliniques cannot be strictly compared. If we consider that 30 per cent. of our patients over 45 years of age have one eye only, the other eye being lost by atrophy, glaucoma, primary or secondary, detachment of retina, etc., one will admit that the figures for Egypt cannot be compared with those of Europe.

Furthermore, when dealing with our patients, it is not always possible to state whether cataract is present or not since the deeper structures of the eye are so frequently obscured by a dense leucoma. It is also true that cases of cataract frequently do not come for treatment at all, in spite of the free treatment provided at the many ophthalmic hospitals which have been founded in Egypt during the last quarter of a century.* At the time when Dr. Osborne made his observations there were fewer hospitals in the country and eye diseases complicating and obscuring cataract were much more common. I believe, therefore, that cataract is actually as common here as it is in Europe although we have less frequent opportunity of operating for it. To make up for this, however, cataract extractions offer us, in at least 90 per cent. of the cases, many surgical difficulties by reason of the complications. Operation, therefore, becomes a very delicate performance and demands on the part of the surgeon much experience, and from the patient constant willingness and untiring patience.

The first complications that the surgeon meets with are the constant occurrence of trachoma, superadded conjunctival infections, lacrymal complications and certain cicatricial lesions such as trichiasis, symblepharon, etc.

The eyelids, even in elderly people, are not always completely free from trachomatous granulations which at one time may be rough, at another time degenerated into small cysts more or less purulent and hidden under the mucous membrane or in the upper fornix. Certain forms of chronic keratitis found in trachomatous patients also arise from these causes. From the same causes also arise the mild cases of iritis which appears five or six days after operation and which delays the cure and provokes secondary cataract. We must, therefore, search for these hidden granulations and destroy them thoroughly.

In the cases of trachoma treated by simple expression and repeated curettage, there results a slight cicatricial contraction of the mucous membrane; but this does not introduce any great difficulty in cataract extraction.

After tarsectomy, however, an operation which is quite

* See the works and statistics of MacCallan up till 1924 and also the statistics of his successors in the Egyptian Public Service. 
frequently performed, there may follow intense scarring in the tarsal conjunctiva to the extent of markedly obliterating the superior fornix. This complication is exceedingly troublesome at the time of operation and for this reason tarsectomy is an operation which should never be performed save under exceptional circumstances.

The chronic forms of blepharo-conjunctivitis often remain rebellious to the most thorough treatment and if incompletely cured are a grave source of post-operative infection. I have seen an operation for cataract followed by a violent iritis as a result of the patient's refusing to await a complete cure of the eyelids before operation. This case, too, is far from constituting the exception. Moreover, even after prolonged antiseptic measures, the eye operated upon remains congested longer than should ordinarily be the case and the dressings are always soiled with an abundant secretion. In these cases we advise that the occlusive dressings should be discontinued as early as possible.

Stenosis of the lacrymal passages and dacryocystitis are not rare and in these cases it is not sufficient to secure the patency of the naso-lacrymal duct, the infecting organisms must be destroyed by antiseptic treatment of long duration and the lacrymal sac itself must be extirpated.

I remember a case, an elderly Italian lady, who had a bilateral dacryocystitis associated with a double cataract. After three months treatment by probing and antiseptics, an extraction of cataract was done in the right eye and within 48 hours after operation there was a fulminating panophthalmitis; but before undertaking to extract the lens of the left eye the left lacrymal sac was removed. Three and a half months later the extraction was done and this time an excellent result was obtained.

Entropion with trichiasis is almost as common as trachoma itself, but our young colleagues are perfectly familiar with the various operations designed to correct this condition and they reject those methods which are likely to lead to shortening of the eyelids. In all plastic operations on the eyelids it is of the greatest importance to preserve the conjunctiva.* One often sees, however, amongst those patients who come for cataract operation, cases suffering also from lagophthalmos and obliteration of the upper fornix and this greatly restricts the field of operation.

Phimosis of the palpebral fissure is another very troublesome complication; in six patients out of ten we are obliged to do a canthotomy or a canthoplasty before being able to insert the lid speculum.

More unpleasant also, if not more serious at the time of operation, is the existence of symblepharon. When it is a matter of

* MacCallan and Péretz.- " The Surgical Treatment of Trichiasis and Entropion of Trachomatous Origin. (Revue du Trachome, September, 1925). 
only a few fine adhesions it is easy to divide them but more often we have to deal with a marked obliteration of either or both fornices. In such cases it is probably better to employ an ordinary lid retractor, instead of using a speculum, but in spite of that the operation is really just as difficult since it is almost impossible to make a satisfactory section through the limbus.

When we come to eonsider the globe itself we find that there are also a great many complicating factors. Some of these make the operation a very difficult one, others by reason of their gravity seriously reduce the chances of a successful result.

In many cases, as a result of previous conjunctivitis and the caustics used so freely in treatment, the bulbar conjunctiva is xerotic, friable and adherent to the episcleral tissues. It bleeds freely when gripped with fixation forceps and it can only be dissected with difficulty. It is, therefore, under such circumstances, wise to use a small pair of forceps with fine teeth and rounded points thus allowing the globe to be held without injury. I confess I still have to learn the best way of dissecting such conjunctiva, as it appears to be associated with insurmountable difficulties.

$\mathrm{W}^{\prime}$ ith regard to the cornea it is the usual thing to find old infiltrations or opacities of varying sizes and often one has to deal with a broad leucomatous pannus perhaps three or four millimetres wide, or a cicatricized trachomatous cornea with an opaque and pitted limbus. It is a very common occurrence for one to have to make the corneal section without being able to see the knife traversing the anterior chamber and that in spite of bright illumination of the field of operation. If the anterior chamber is shallow, we do a preliminary iridectomy so that the anterior chamber will afterwards be reformed to such an extent as to permit one to make the section more easily. Further, we recommend the use of a short narrow-bladed Graefe's knife, for, the field of operation being usually very restricted, one is very apt with the ordinary Graefe's knife to puncture the skin of the internal canthus or the free border of the upper lid.

It is our practice always to do an iridectomy, for, considering the conditions, it is without doubt the method of choice in Egypt. To preserve a round pupil in the ordinary extracapsular extraction is to court almost certain secondary glaucoma.

Egypt is truly the country of chronic glaucoma. The statistic's, which are most sriking, show that amongst aged patients 40 per cent. are glaucomatous. It is, therefore, possible that every case of cataract may in the course of its evolution be complicated by a more or less insidious glaucoma. The oculist, therefore, must not fail to remember this fact when he is dealing with a case of cataract.

The fear of glaucoma is the beginning of wisdom. It is conse- 
quently wise never to instil atropine, in a case of cataract unless the tension can be controlled by the tonometer. Usually, two drops of $1 / 2$ per cent. homatropine or one drop of 4 per cent. cocaine will dilate the pupil sufficiently to enable a thorough examination of the lens and the fundus to be made.

Another precaution which should be taken is never to allow a case with incipient cataract to remain for too long a period without being examined. In Europe it is common to leave patients of this description for three or six months without anxiety but in Egypt we would be taking too great a risk in following such a practice. Were we to do so we might possibly in the meantime allow some eyes to be lost as a result of glaucoma. For all these reasons, therefore, I cannot insist too strongly on the necessity of watching such cases very carefully and also of doing a preliminary iridectomy every time there is a possibility of glaucoma. One can appreciate, therefore, more easily why iridectomy must be done in every case of extraction of cataract.

We have observed elsewhere that the lens is prematurely cataractous in some patients who have been operated upon for chronic glaucoma and that it gives rise to a type of early. glaucomatous cataract. The co-existence of glaucoma and of cataract, very rare in Europe, is of the commonest occurrence in Egypt. This, however, is really outside the subject.*

We frequently have to operate for cataract complicated with glaucoma and indeed we are often obliged to do so even when the light projection is only fair. These patients after their operation and in spite of their limited field of vision can go about and also carry on their house-work for many years. As instances of this I may quote the following cases :-

Ahmed Qh. Bey, aged 66 years, lost the sight of his right eye during infancy. In 1916, an iridectomy for chronic glaucoma in the left eye was done. Later, he developed cataract with bad projection of light. He went to several oculists all of whom declined to extract the cataract, but at last a colleague newly arrived in the city, who was more bold performed the operation. The extraction with another iridectomy was done in 1921 and the result was entirely successful. L.V.with $\frac{+10}{+} 4$ D. sph. $110^{\circ}=\frac{6}{12}$ Six years later the patient had practically the same acuity of vision.

Mme. S. K., aged 70 years. The right eye on examination was found to be lost as a result of glaucoma absolutum. The left showed cicatrized trachoma, leucomatous pannus, central nebula of the cornea, chronic glaucoma and cataract. The light projection of this eye was also defective. In spite of this an iridectomy

* The author dealt with the question of glaucomatous cataract at the 13th International Congress of Ophthalmology, at Amsterdam, September, 1929. 
for the glaucoma was first done and after six months the cataract was extracted giving the patient $\mathrm{V}$. with $+12 \mathrm{D}$. $\mathrm{sph} .=6 / 36$. The patient was delighted at being able to return to her household duties.

In addition to glaucoma, other ocular affections which complicate cataract are high myopia and retinal arterio-sclerosis.

Myopia in Egyptians is a hereditary disease. This fact was pointed out in the first place by Jacovides and by MacCallan, but it has been more carefully studied by Meyerhof in 1914.* In 1908, MacCallan found 26 per cent. of myopes amongst pupils of a certain public school, while Meyerhof found 32 per cent. of males and 16 per cent. of females affected with myopia. Jacovides in Alexandria has recorded myopia in 60 per cent. of his patients.

Whatever may be the reason, cataract amongst the myopes of Egypt assumes an aspect specially characterized by the slow onset of maturity, liquefaction of the vitreous and fragility of the zonular ligament. Such a condition is a terror to the surgeon and compels him to adopt a procedure which may be summed up in the following words: "Traumatize the cataract in myopia as little as possible." If the nucleus is small we do a linear extraction through a limbic section using a keratome after a small conjunctival flap has first been dissected up. In this way one ought almost certainly to be able to avoid loss of vitreous. A retrobulbar injection of novocaine with adrenalin, made 10 minutes before operation. also makes the operation more safe by securing perfect anaesthesia of the iris and lowering of the intra-ocular tension.

Even for patients with only one eye we are frequently called upon to operate for cataract and that, too, when there may be present such complicating factors as retinal arterio-sclerosis and even detachment of the retina. Extraordinary as it may appear to operate in such cases, operation often procures some satisfaction for the patient even though it be sometimes more moral than material.

In conclusion I may add that my early statistics did not show the same happy results in summer as they did in winter. This is undoubtedly due to the fact that the microbic flora of the conjunctiva find in the heat and the humidity of the conjunctival sac, especially when it is closed by a dressing, just the very conditions favourable for rapid multiplication. Experience has, therefore, since taught me not to operate for cataract during the hottest months of the year, i.e., from June to September, when the temperature in Cairo ranges between $98^{\circ}$ and $104^{\circ} \mathrm{F}$.

* Meyerhof - A Study on Myopia as a racial and hereditary disease. (Annales d'Oculistique, April, 1914.) 